\title{
Global linear instability of rotating-cone boundary layers in a quiescent medium
}

\author{
Christian Thomas* \\ School of Mathematical Sciences, Monash University, Clayton, Melbourne, Victoria 3800, Australia \\ Christopher Davies \\ School of Mathematics, Cardiff University, Cardiff CF24 4AG, United Kingdom
}

(Received 9 September 2018; published 8 April 2019)

\begin{abstract}
The global linear stability of the family of infinite rotating-cone boundary layers in an otherwise still fluid is investigated using a velocity-vorticity form of the linearized NavierStokes equations. The formulation is separable with respect to the azimuthal direction. Thus, disturbance development is simulated for a single azimuthal mode number. Numerical simulations are conducted for an extensive range of cone half-angles $\left(\psi \in\left[20^{\circ}: 80^{\circ}\right]\right)$ and stability parameters (Reynolds number, azimuthal mode number), where conditions are taken to be near those specifications necessary for the onset of absolute instability. A localized impulsive wall forcing is implemented that excites disturbances that form wave packets. This allows the disturbance evolution to be traced in the spatial-temporal plane. When a homogeneous flow approximation is utilised that neglects the spatial variation of the basic state, linear perturbations display characteristics consistent with local stability theory. For disturbances to the genuine spatially dependent inhomogeneous flow, global linear instability characterized by a faster than exponential temporal growth arises for azimuthal mode numbers greater than the conditions for critical absolute instability. Furthermore, a reasonable prediction for the azimuthal mode number needed to bring about a change in global behavior is achieved by coupling solutions of the GinzburgLandau equation with local stability properties. Thus, the local-global stability behavior is qualitatively similar to that found in the infinite rotating-disk boundary layer and many other globally unstable flows.
\end{abstract}

DOI: 10.1103/PhysRevFluids.4.043902

\section{INTRODUCTION}

Current interest in laminar-turbulent transition in rotating boundary layers was motivated by the discovery by Lingwood [1] that the von Kármán [2] flow that develops on an infinite rotatingdisk is locally absolutely unstable. Lingwood undertook a local linear stability analysis, whereby a homogeneous flow approximation was implemented that removed the radial dependence of the basic state. Absolute instability was then identified via the Briggs [3] pinch-point method and is formed following the coalescence of two modes: crossflow [4] and a spatially damped mode [5]. Following Lingwood's [1] revelations on the infinite rotating-disk, several further case studies were undertaken that examined the local stability properties of the larger family of rotating boundary layers. Garrett and Peake [6-8] applied local linear stability theory to both rotating-sphere and rotating-cone boundary layers, within a still and a uniform axial flow. Regions of absolute instability were discovered within the respective stability parameter spaces. Furthermore, a uniform axial flow

*christian.thomas@monash.edu 
was found to delay the onset of absolute instability to larger Reynolds numbers than those obtained for the unmodified flow.

The global linear stability of the radially dependent von Kármán flow was first examined by Davies and Carpenter [9] using a velocity-vorticity [10] form of the linearized Navier-Stokes equations. Disturbance development to an impulsive wall forcing was numerically simulated for a range of radial values and azimuthal mode numbers near the critical conditions for absolute instability. However, for those parameter settings studied, the absolutely unstable mechanism was not sufficient to excite any sustained temporally growing oscillations that might be associated with a global linear instability. Instead the long-term disturbance response was governed by convective characteristics.

Recent studies on the rotating-disk boundary layer by Appelquist et al. have examined nonlinearity [11] and finite disk effects [12-14]. The latter strategy was implemented to provide an improved representation of experimental models. It was found that globally unstable disturbances could be created by imposing conditions at the outer boundary of the disk that account for downstream turbulence. However, for a disk of infinite radius, Appelquist et al. [13,14] confirmed the earlier results of Davies and Carpenter [9] and were only able to find disturbance development matching to global stability.

Despite earlier suggestions that the infinite rotating-disk boundary layer was globally stable, a recent numerical investigation by Thomas and Davies [15] has shown that, for azimuthal mode numbers greater than that corresponding to critical absolute instability (that had been the subject of the earlier investigations $[9,11,13,14]$ ), linear disturbances in the infinite rotating-disk exhibit globally unstable behavior. Although for the time period that disturbance evolution could be accurately simulated, temporal frequencies again varied in both space and time, and no fixed global mode was realized. Furthermore, the Reynolds number associated with these high azimuthal mode numbers coincides with the mid to upper range of values reported experimentally for transition [16-18]. Thus, the absolutely unstable mechanism discovered by Lingwood [1] is, for large enough azimuthal mode numbers, sufficient to engineer a form of global linear instability in the von Kármán flow on an infinite rotating-disk. Hence, the local-global stability characteristics of the infinite rotating-disk boundary layer are similar to the description given by Huerre and Monkewitz [19] that states a region of local absolute instability is a necessary but not sufficient condition for global instability to ensue.

Davies and co-workers [15,20-23] coupled solutions of the linearized Ginzburg-Landau equation [24] with numerical simulation results and showed that the global linear stability of the infinite rotating-disk is governed by a detuning effect [25-27]. Depending on the precise balance between the radial variations in the temporal frequency and matching shifts in growth, it was possible for a flow to remain globally stable even though it might be locally absolutely unstable [19]. Furthermore, Thomas and Davies [15] utilised the Ginzburg-Landau model [24] to predict the azimuthal mode number required for global linear instability, based only on those disturbances to the approximate radially homogeneous flow.

Applications of rotating-cones include those flows that develop around nose cones in aircraft engines and spinning projectiles. Early experimental investigations on rotating-cone boundary layers were limited to measurements of transition to turbulence [28-31]. Corotating spiral vortices were found to form along the surface of the rotating-cone [32-34], which were similar in appearance to those discovered by Gregory et al. [4] on the rotating-disk. Theoretical studies on the convective instabilities associated with the spiral vortices were undertaken by Kobayashi and co-workers [32,35]. Using a new base flow model, Garrett et al. [36] improved upon the earlier linear stability calculations presented by Garrett and Peake [8] for rotating-cones in a uniform axial flow. However, results were unchanged for those boundary layers with a zero axial flow. Further investigations on the family of rotating-cone boundary layers were undertaken by the Garrett group [37-40], who studied the type of convective instabilities that develop for variable cone half-angles. For broad rotating-cones, the crossflow instability that forms the corotating vortices on the rotating-disk was found to dominate the boundary layer stability process (at least until the conditions required 


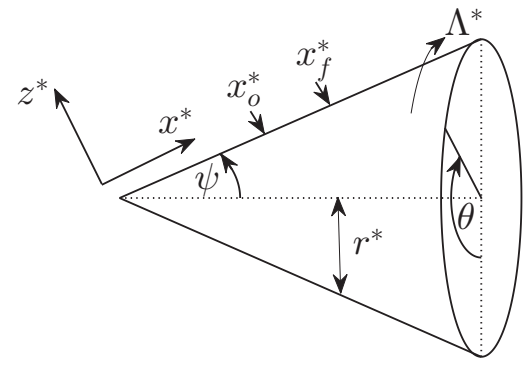

FIG. 1. Illustration of the rotating-cone geometry.

to establish absolute instability were met). However, for slender rotating-cones, asymptotic and numerical methods located a new Görtler-type instability that appears as counter-rotating spiral vortices and is governed by a centrifugal mechanism.

For the current study, the velocity-vorticity formulation developed by Davies and Carpenter [10] and the Ginzburg-Landau model [24] utilized by Davies and co-workers [15,20-22] are applied to the family of infinite rotating-cone boundary layers that develop in a quiescent medium. Our analysis will focus on the global linear stability of disturbances to an impulse excitation and the variation in behavior as the azimuthal mode number is increased to larger values. The primary aim of this paper is then to demonstrate that the findings of Thomas and Davies [15] on global linear instability, and indeed that found in many other globally unstable flows [19], are universal to rotating boundary layers.

In the subsequent sections the evolution of linear disturbances to the family of rotating-cone boundary layers in an otherwise still fluid is investigated for an extensive range of cone half-angles and azimuthal mode numbers. Furthermore, the rotating-cone is assumed to be infinite along the streamwise direction. In Sec. II the governing equations for generating the basic state (based on the method presented by Garrett and Peake [8]) on a rotating-cone and the velocity-vorticity formulation for simulating the evolution of perturbations are described. The Ginzburg-Landau model developed by Hunt and Crighton [24] and utilized by the Davies group [15,20-23] is applied to the family of infinite rotating-cone boundary layers to predict the azimuthal mode number needed to bring about a change in the global behavior, from stable to unstable. Disturbance development to the genuine spatially dependent inhomogeneous flow is then numerically simulated for an extensive range of rotating-cone half-angles and flow settings. Finally, conclusions are presented in Sec. V.

\section{FORMULATION}

\section{A. Undisturbed flow}

In a fixed orthogonal curvilinear coordinate system $\left\{x^{*}, \theta, z^{*}\right\}$, a rigid cone with circular cross section and half-angle $\psi$, rotates about its axis of symmetry with a constant angular frequency $\Lambda^{*}$ in an otherwise still fluid (as illustrated in Fig. 1). The cross-sectional radius of the cone $r^{*}=x^{*} \sin \psi$, while the streamwise and wall-normal directions are assumed to be semi-infinite: $0 \leqslant x^{*}<\infty$ and $0 \leqslant z^{*}<\infty$. The origin $\left\{x^{*}, z^{*}\right\}=\{0,0\}$ is located at the apex of the cone. The undisturbed flow field associated with this particular coordinate system is denoted by the dimensional vector

$$
\mathbf{U}^{*}=\left\{U_{x}^{*}, U_{\theta}^{*}, U_{z}^{*}\right\}
$$

where an asterisks represents dimensional quantities. As the cone rotates within a fixed frame of reference, Coriolis force terms are omitted from all subsequent equations. Thus, numerical solutions are more easily compared with the theoretical analysis of Garrett and Peake [8], who implemented a similar treatment of the Coriolis force terms in their study. 
The nondimensional steady basic state is obtained by introducing a von Kármán [2] type similarity substitution,

$$
\left\{U_{x}^{*}, U_{\theta}^{*}, U_{z}^{*}\right\}=\left\{x^{*} \Lambda^{*} \sin \psi F(z), x^{*} \Lambda^{*} \sin \psi G(z), \delta^{*} \Lambda^{*} H(z)\right\},
$$

where the wall-normal functions $F, G$, and $H$ denote the velocity profiles along the respective streamwise, azimuthal, and wall-normal directions. The streamwise and wall-normal axes are then dimensionalized on the length scale $\delta^{*}=\left(v^{*} / \Lambda^{*}\right)^{\frac{1}{2}}$, where $v$ represents the kinematic viscosity of the fluid: $x=x^{*} / \delta^{*}$ and $z=z^{*} / \delta^{*}$. Additionally, the circumferential speed of the rotating-cone about a streamwise reference position $x_{o}^{*}$ is used to nondimensionalize the velocity field. Thus, the nondimensional representation of the undisturbed flow on the rotating-cone is given as

$$
\mathbf{U}_{\mathbf{B}}(x, z, \psi)=\left(\frac{x \sin \psi}{\operatorname{Re}} F(z), \frac{x \sin \psi}{\operatorname{Re}} G(z), \frac{1}{\operatorname{Re}} H(z)\right),
$$

where the Reynolds number is defined as

$$
\operatorname{Re}=\frac{x_{o}^{*} \Lambda^{*} \delta^{*} \sin \psi}{\nu^{*}}=\frac{x_{o}^{*} \sin \psi}{\delta^{*}}=x_{o} \sin \psi .
$$

On substituting the similarity variables (1) into the Navier-Stokes equations for curvilinear coordinates [41], the following system of ordinary differential equations is obtained:

$$
\begin{gathered}
\left(F^{2}-G^{2}\right) \sin \psi+F^{\prime} H=F^{\prime \prime}, \\
2 F G \sin \psi+G^{\prime} H=G^{\prime \prime}, \\
2 F \sin \psi+H^{\prime}=0,
\end{gathered}
$$

where a prime denotes differentiation with respect to $z$. This particular system of equations is similar to that derived by von Kármán [2] for the rotating-disk boundary layer, in the instance that $\psi=90^{\circ}$. Equations (4a)-(4c) are solved (using routines available within MATLAB) subject to the boundary conditions on the cone surface

$$
F=G-1=H=0 \quad \text { on } \quad z=0
$$

and the freestream conditions

$$
F \rightarrow 0, \quad G \rightarrow 0 \quad \text { as } \quad z \rightarrow \infty
$$

Figure 2 displays the steady velocity flow profiles $F, G$, and $H$ for four cone half-angles $\psi \in$ $\left[20^{\circ}: 80^{\circ}\right]$ at intervals $\Delta \psi=20^{\circ}$.

\section{B. Velocity-vorticity formulation}

The global linear stability of perturbations to the system of infinite rotating-cone boundary layers was examined by deriving a velocity-vorticity formulation, similar to that utilized in the earlier studies on the infinite rotating-disk $[9,10,15]$. Total velocity and vorticity fields are respectively defined as

$$
\mathbf{U}=\mathbf{U}_{\mathbf{B}}+\mathbf{u}, \quad \Omega=\boldsymbol{\Omega}_{\mathbf{B}}+\omega,
$$

where $\mathbf{U}_{\mathbf{B}}$ is given by (2) and the undisturbed vorticity $\boldsymbol{\Omega}_{\mathbf{B}}=\nabla \times \mathbf{U}_{\mathbf{B}}$. Perturbation variables are denoted as

$$
\mathbf{u}=\left(u_{x}, u_{\theta}, u_{z}\right), \quad \boldsymbol{\omega}=\left(\omega_{x}, \omega_{\theta}, \omega_{z}\right),
$$


(a)

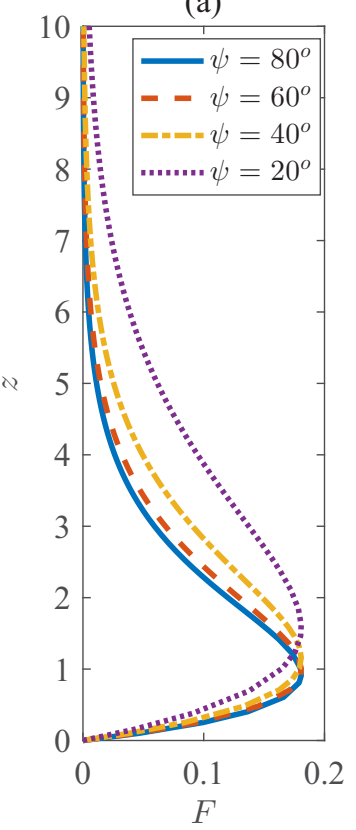

(b)

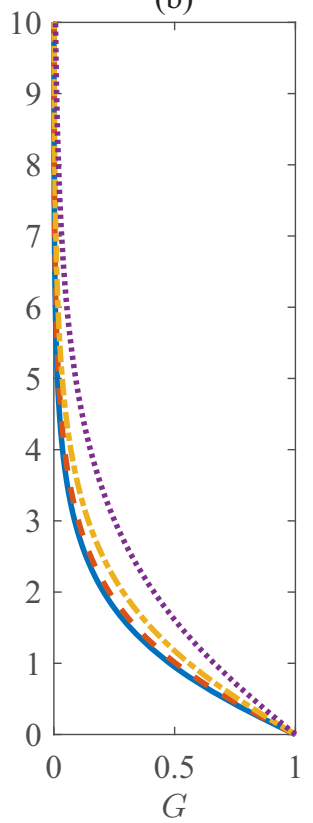

(c)

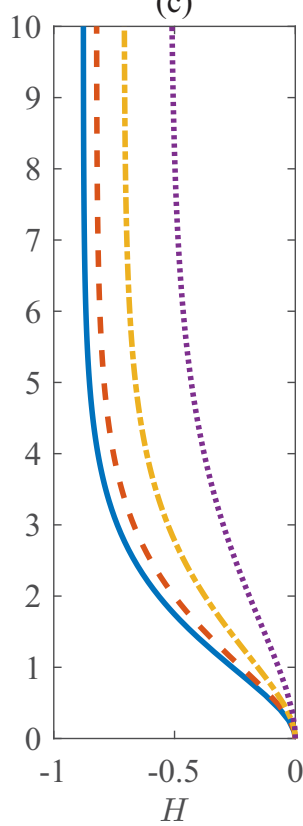

FIG. 2. Steady velocity flow profiles for four cone half-angles. (a) $F$, (b) $G$, (c) $H$.

which are separated into primary $\left\{\omega_{x}, \omega_{\theta}, u_{z}\right\}$ and secondary components $\left\{u_{x}, u_{\theta}, \omega_{z}\right\}$. The three primary fields are given as solutions of the linearized governing equations

$$
\begin{gathered}
\frac{\partial \omega_{x}}{\partial t}+\frac{1}{h}\left\{\frac{\partial N_{z}}{\partial \theta}-\frac{\partial\left(h N_{\theta}\right)}{\partial z}\right\}=\frac{1}{\operatorname{Re}}\left\{\nabla^{2} \omega_{x}-\frac{\sin \psi}{h^{2}}\left(\omega_{x} \sin \psi+\omega_{z} \cos \psi\right)-\frac{2 \sin \psi}{h^{2}} \frac{\partial \omega_{\theta}}{\partial \theta}\right\}, \\
\frac{\partial \omega_{\theta}}{\partial t}+\frac{\partial N_{x}}{\partial z}-\frac{\partial N_{z}}{\partial x}=\frac{1}{\operatorname{Re}}\left\{\left(\nabla^{2}-\frac{1}{h^{2}}\right) \omega_{\theta}+\frac{2}{h^{2}} \frac{\partial}{\partial \theta}\left(\omega_{x} \sin \psi+\omega_{z} \cos \psi\right)\right\}, \\
\nabla^{2} u_{z}=\frac{1}{h}\left(\frac{\partial \omega_{x}}{\partial \theta}-\frac{\partial\left(h \omega_{\theta}\right)}{\partial x}\right),
\end{gathered}
$$

where

$$
\begin{gathered}
h=x \sin \psi+z \cos \psi \\
\nabla^{2}=\frac{\partial^{2}}{\partial x^{2}}+\frac{\sin \psi}{h} \frac{\partial}{\partial x}+\frac{1}{h^{2}} \frac{\partial^{2}}{\partial \theta^{2}}+\frac{\partial^{2}}{\partial z^{2}}+\frac{\cos \psi}{h} \frac{\partial}{\partial z}
\end{gathered}
$$

and

$$
\mathbf{N}=\left(N_{x}, N_{\theta}, N_{z}\right)=\boldsymbol{\Omega}_{\boldsymbol{B}} \times \mathbf{u}+\boldsymbol{\omega} \times \mathbf{U}_{\mathbf{B}} .
$$

Equations (5a) and (5b) represent the streamwise and azimuthal components of the vorticity transport equation in curvilinear coordinates, while $(5 \mathrm{c})$ is the wall-normal component of the Poisson equation. The three secondary variables are defined directly in terms of the primary variables using explicit formula that are derived from the definitions for vorticity and the solenoidal condition:

$$
\omega=\nabla \times \mathbf{u} \text { and } \nabla \cdot \omega=0 .
$$


Boundary constraints are then formulated in the manner outlined by Davies and Carpenter [10], where the no-slip conditions are replaced with integral conditions imposed on the primary variables.

For the subsequent global linear stability investigation, perturbations are assumed to be periodic with respect to the azimuthal direction:

$$
\{\mathbf{u}, \boldsymbol{\omega}\}=\{\hat{\mathbf{u}}, \hat{\boldsymbol{\omega}}\} e^{i n \theta},
$$

where $n=\beta \operatorname{Re}$ is the integer valued azimuthal mode number and $\beta$ is the azimuthal wavenumber that is more commonly used to indicate regions of linear instability in studies that have implemented a homogeneous flow approximation. No other assumptions are made about the form of the perturbations.

In local linear stability analysis that applies a homogeneous flow approximation to the basic state [1,8], the variable $h$ in (5d) is simplified, by assuming that $z / x \sin \psi \ll 1$. Additionally, the $x$-dependence of the base flow (2) is removed by setting $x=x_{o}$. Thus, the scale factor $h$ is set equal to Re. However, for the subsequent study, the homogeneous flow approximation is only imposed in those cases used to validate the numerical simulation results; refer to §II.C. For the main numerical computations presented in $\S \mathrm{IV}$, disturbance development is simulated to the inhomogeneous flow where the streamwise $x$-dependence is retained.

Discretization of the governing Eqs. (5) was achieved using the scheme outlined in Davies and Carpenter [10]. A fourth-order centered, compact finite difference method was implemented along the streamwise $x$-direction and a Fourier spectral expansion (outlined above) was used in the azimuthal direction. The wall-normal $z$-direction was treated using a Chebyshev spectral series representation, with coordinates carefully mapped from the semi-infinite domain onto a finite interval. Finally, time-marching was achieved using semi-implicit methods. Further details of the numerical formulation are presented in Davies and Carpenter [10].

\section{Code validation}

Perturbations were impulsively excited through a localised streamwise forcing that was implemented via a linearisation of the no-slip conditions on the cone surface. The impulsive forcing was centered about a streamwise position $x_{f}$ and was applied for a sufficient time duration to excite a broad range of stationary and traveling perturbations. Eventually the strongest growing perturbations form spatial-temporal wave packets, which can be used to draw conclusions regarding the global linear stability of the system of rotating-cone boundary layers.

Table I compares the critical conditions for absolute instability given by Garrett and Peake [8] with those results obtained via numerical computations based on a homogeneous flow approximation (bold font). Temporal frequencies and growth rates were computed using the complex-valued quantity

$$
f=\frac{i}{\omega_{\theta, w}} \frac{\partial \omega_{\theta, w}}{\partial t}
$$

where $\omega_{\theta, w}$ is the azimuthal vorticity perturbation on the cone surface. The real and imaginary parts of $f$ define the respective temporal frequency and growth rate of the disturbance at each point in space and time. To avoid expensive computational costs, numerical simulations were carried out for integer valued $\mathrm{Re}$ and $n$ only. Although this inevitably introduces some minor variations in the critical parameters, the computations tabulated in Table I are still in excellent agreement with those results presented in Garrett and Peake [8]. In most instances, both $f_{c}$ and the streamwise wave number $\alpha_{c}$ (which was determined using a similar formula to that defined above) are identical to within two or three decimal places. Additionally, frequency calculations (in Table I and in the subsequent discussion) are presented as $g=f \mathrm{Re}$, where a multiplicative factor of the Reynolds number has been introduced to help draw comparisons between numerical simulations based on the homogeneous and inhomogeneous flows. 
TABLE I. Critical values for the onset of absolute instability as found by Garrett and Peake [8] using a localized stability analysis with a homogeneous flow approximation. Values given in bold are determined from numerical simulations using the same approximation.

\begin{tabular}{lccclcr}
\hline \hline$\psi$ & $\mathrm{Re}_{a}$ & $\beta_{a}$ & $n_{a}$ & $f_{a}$ & $\alpha_{a}$ & $g_{a}$ \\
\hline $20^{\circ}$ & 289.95 & 0.080 & 23 & 0.059 & $0.375-i 0.208$ & 17.11 \\
$\mathbf{2 0}^{\circ}$ & $\mathbf{2 9 0}$ & $\mathbf{0 . 0 8 0}$ & $\mathbf{2 3}$ & $\mathbf{0 . 0 5 9 2}$ & $\mathbf{0 . 3 7 3}-\boldsymbol{i 0 . 2 0 6}$ & $\mathbf{1 7 . 1 7}$ \\
$30^{\circ}$ & 353.47 & 0.096 & 34 & 0.071 & $0.309-i 0.172$ & 25.10 \\
$\mathbf{3 0}^{\circ}$ & $\mathbf{3 5 3}$ & $\mathbf{0 . 0 9 6}$ & $\mathbf{3 4}$ & $\mathbf{0 . 0 7 1 2}$ & $\mathbf{0 . 3 1 1}-\boldsymbol{i 0 . 1 6 9}$ & $\mathbf{2 5 . 1 3}$ \\
$40^{\circ}$ & 402.49 & 0.109 & 44 & 0.081 & $0.272-i 0.152$ & 32.60 \\
$\mathbf{4 0}^{\circ}$ & $\mathbf{4 0 3}$ & $\mathbf{0 . 1 0 9}$ & $\mathbf{4 4}$ & $\mathbf{0 . 0 8 0 6}$ & $\mathbf{0 . 2 7 4}-\boldsymbol{i 0 . 1 4 7}$ & $\mathbf{3 2 . 5 0}$ \\
$50^{\circ}$ & 440.60 & 0.118 & 52 & 0.088 & $0.249-i 0.139$ & 38.77 \\
$\mathbf{5 0}^{\circ}$ & $\mathbf{4 4 1}$ & $\mathbf{0 . 1 1 8}$ & $\mathbf{5 2}$ & $\mathbf{0 . 0 8 7 5}$ & $\mathbf{0 . 2 5 0}-\boldsymbol{i 0 . 1 3 5}$ & $\mathbf{3 8 . 5 9}$ \\
$60^{\circ}$ & 469.42 & 0.126 & 59 & 0.093 & $0.234-i 0.131$ & 43.66 \\
$\mathbf{6 0}^{\circ}$ & $\mathbf{4 7 0}$ & $\mathbf{0 . 1 2 6}$ & $\mathbf{5 9}$ & $\mathbf{0 . 0 9 3 3}$ & $\mathbf{0 . 2 3 5}-\boldsymbol{i 0 . 1 2 6}$ & $\mathbf{4 3 . 8 5}$ \\
$70^{\circ}$ & 489.78 & 0.131 & 64 & 0.097 & $0.224-i 0.125$ & 47.51 \\
$\mathbf{7 0}^{\circ}$ & $\mathbf{4 9 0}$ & $\mathbf{0 . 1 3 1}$ & $\mathbf{6 4}$ & $\mathbf{0 . 0 9 7 0}$ & $\mathbf{0 . 2 2 3}-\boldsymbol{i 0 . 1 2 1}$ & $\mathbf{4 7 . 5 3}$ \\
$80^{\circ}$ & 502.12 & 0.134 & 67 & 0.099 & $0.219-i 0.122$ & 49.71 \\
$\mathbf{8 0}^{\circ}$ & $\mathbf{5 0 2}$ & $\mathbf{0 . 1 3 4}$ & $\mathbf{6 7}$ & $\mathbf{0 . 0 9 9 2}$ & $\mathbf{0 . 2 2 0}-\mathbf{i 0 . 1 1 8}$ & $\mathbf{4 9 . 8 0}$ \\
$90^{\circ}$ & 507.32 & 0.135 & 68 & 0.099 & $0.217-i 0.122$ & 50.22 \\
$\mathbf{9 0}^{\circ}$ & $\mathbf{5 0 8}$ & $\mathbf{0 . 1 3 5}$ & $\mathbf{6 8}$ & $\mathbf{0 . 1 0 0 0}$ & $\mathbf{0 . 2 1 8}-\boldsymbol{i 0 . 1 1 7}$ & $\mathbf{5 0 . 8 0}$ \\
\hline \hline
\end{tabular}

\section{PREDICTING GLOBAL INSTABILITY VIA THE GINZBURG-LANDAU EQUATION}

Thomas and Davies [15,21] utilized solutions of the linearized Ginzburg-Landau equation [24] to provide an explanation for why disturbances in the infinite rotating-disk boundary layer can become globally unstable or remain stable. Parameters of the Ginzburg-Landau equation were carefully matched to disturbance properties of the numerical simulations, allowing Thomas and Davies to interpret the stability characteristics and predict the critical azimuthal mode number $n_{c}$, whereby for $n>n_{c}$ global instability ensues. The same procedure will be utilized here to predict the azimuthal mode numbers $n_{c}$ needed to bring about global linear instability in the family of infinite rotating-cone boundary layers. However, instead of repeating the detailed description of the method presented in Thomas and Davies $[15,21]$ we will only outline the salient points of the strategy here.

The linearized Ginzburg-Landau equation is given as

$$
\frac{\partial A}{\partial t}+U \frac{\partial A}{\partial x}=\mu A+\gamma \frac{\partial^{2} A}{\partial x^{2}}
$$

where $A(x, t)$ is a measure of the disturbance amplitude at the spatial location $x$ and time $t$. The parameters $\mu, U$, and $\gamma$ denote the stability, flow convection, and diffusion/dispersion effects, respectively. If $\mu$ is allowed to vary linearly with the spatial direction,

$$
\mu(x)=\mu_{0}+\mu_{1} x
$$

where the real and imaginary parts of $\mu_{1}$ represent the respective spatial variations in the temporal growth rate and matching frequency, the following impulse Green's solution to (8) can be derived $[21,24]:$

$$
G(x, t)=\sqrt{\frac{1}{4 \pi \gamma t}} \exp \left(\frac{1}{2} \mu_{1} x t+\frac{1}{12} \mu_{1}^{2} \gamma t^{3}-\frac{x^{2}}{4 \gamma t}\right) \exp \left\{i\left(\alpha_{0} x-f_{0} t\right)\right\}
$$


where

$$
f_{0}=i\left(\mu_{0}-\frac{U^{2}}{4 \gamma t}\right) \quad \text { and } \quad \alpha_{0}=-i \frac{U}{2 \gamma} .
$$

The temporal frequency $f_{0}$ and radial wave number $\alpha_{0}=\alpha_{0, r}+i \alpha_{0, i}$ are both complex, while the spatial coordinate $x$ is shifted, so that the impulse is centered about $x=0$. In the instance that $\mu_{1}=0$, the imaginary part of $\mu_{0}$ represents the temporal growth rate that determines whether or not the flow is stable, convectively unstable, or absolutely unstable.

The complex temporal frequency of the Green's solution (9a) can be determined using (7) (by replacing $\omega_{\theta, w}$ with $G$ ) to give the disturbance growth rate

$$
\operatorname{Re}(f) \rightarrow \rho t^{2} \quad \text { as } \quad t \rightarrow \infty \quad \text { for } \quad \rho=\left[\left(\mu_{1, r}^{2}-\mu_{1, i}^{2}\right) \gamma_{r}-2 \mu_{1, r} \mu_{1, i} \gamma_{i}\right]
$$

Thus, for large time $t$ the response of the disturbance is governed by the stability coefficient $\mu_{1}=\mu_{1, r}+i \mu_{1, i}$ and the diffusion/dispersion parameter $\gamma=\gamma_{r}+i \gamma_{i}$. A negative valued $\rho$ will then bring about temporal decay and global stability, while positive $\rho$ corresponds to temporal growth and global instability.

Parameters $\mu_{1}$ and $\gamma$ can be chosen using the procedure outlined in Thomas and Davies [15,21], leading to the expressions

$$
\mu_{1, r}=2 \frac{\partial g_{i}}{\partial x} \quad \text { and } \quad \mu_{1, i}=-2 \frac{\partial g_{r}}{\partial x}
$$

where $g=g_{r}+i g_{i}$ is the complex temporal frequency obtained via numerical simulations of the disturbance development to the homogeneous flow. Meanwhile, dispersion and diffusion effects are given by the relationships

$$
\frac{1}{\gamma}=\frac{\gamma_{r}}{|\gamma|^{2}}-i \frac{\gamma_{i}}{|\gamma|^{2}}
$$

for

$$
\frac{\gamma_{r}}{|\gamma|^{2}}=-\frac{2 \alpha_{0, i}}{U} \quad \text { and } \quad \frac{\gamma_{i}}{|\gamma|^{2}}=\frac{2}{U}\left(\left.\frac{\partial \phi}{\partial x}\right|_{\max }-\alpha_{0, r}\right),
$$

where the convection velocity $U$ is measured about the contour line that the disturbance achieves a maximum amplitude and $\left.\frac{\partial \phi}{\partial x}\right|_{\max }$ represents the corresponding phase shift.

Disturbance development to the homogeneous flow was simulated for an extensive range of parameter settings (i.e., Re and $n$ ) and for cone half-angles $\psi \in\left[20^{\circ}: 90^{\circ}\right]$ at intervals $\Delta \psi=10^{\circ}$. For each azimuthal mode number $n$ investigated, the analysis was performed about the streamwise location (Reynolds number) corresponding to critical absolute instability. The parameters required for computing $\rho$ [recall Eqs. (10)-(12)] were carefully calculated, and solutions are plotted in Fig. 3. Each curve (eight in total) corresponds to a fixed half-angle $\psi$ and represents a measure of the predicted long-term growth as a function of the azimuthal mode number, where $\psi$ increases in size from left to right. The plot on the outer right corresponds to the solution given by Thomas and Davies [15] for the infinite rotating-disk $\left(\psi=90^{\circ}\right)$. Cross markers, located along each curve, indicate the respective azimuthal mode numbers $n_{a}$ for the onset of absolute instability. For each half-angle modeled, a negative valued $\rho$ is obtained for $n=n_{a}$. However, for larger azimuthal mode numbers, a positive valued $\rho$ is eventually achieved. This would suggest that for sufficiently large $n$, unstable behavior is realized for all cone half-angles. The predicted azimuthal mode number $n_{c}$ required for positive $\rho$ and unstable characteristics are documented in Table II. In each instance $n_{c}>n_{a}$ (compare results given in Tables I and II). For example, the above method predicts that for $\psi=40^{\circ}$, global instability will appear for

$$
n \gtrsim n_{c}=51>44=n_{a} .
$$




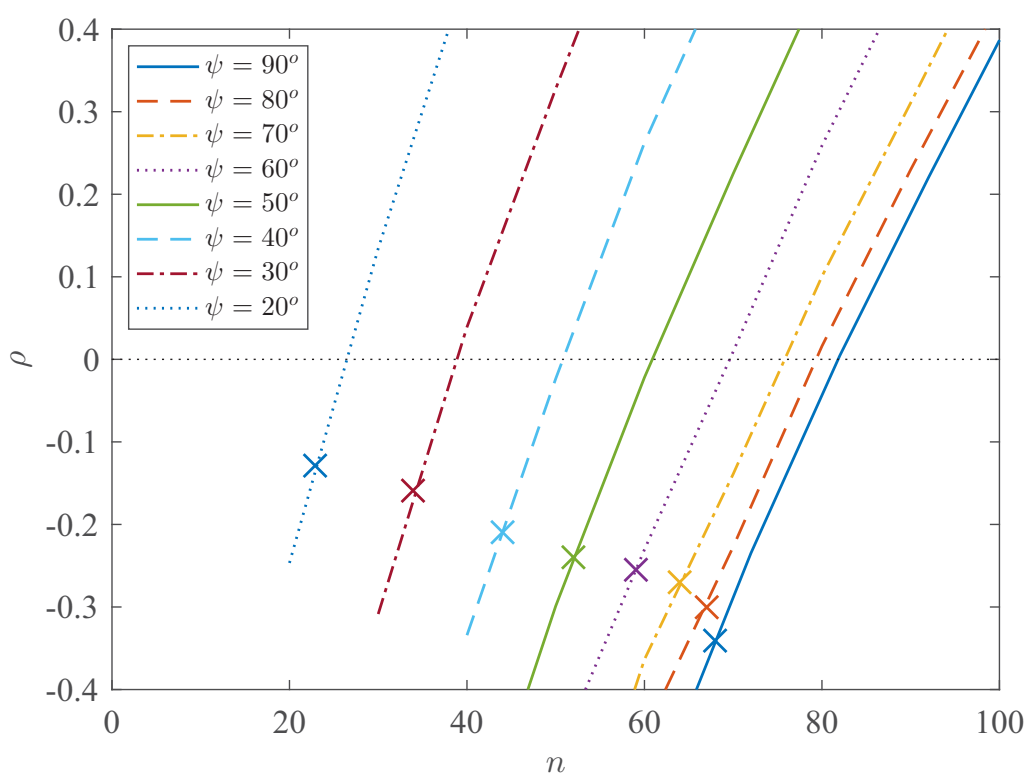

FIG. 3. Growth rates $\rho$ as a function of the azimuthal mode number $n$ for cone half-angles $\psi=\left[20^{\circ}: 10\right.$ : $90^{\circ}$ ]. Cross markers indicate the respective azimuthal mode numbers for critical absolute instability.

For all $n>n_{c}$, strong temporal growth and global instability might be expected to develop in the inhomogeneous flow.

\section{DISTURBANCE DEVELOPMENT IN THE INHOMOGENEOUS FLOW}

The evolution of disturbances to the genuine spatially dependent family of infinite rotating-cone boundary layers was numerically simulated to verify the above predictions for the onset of global instability. We will first illustrate and describe the type of behavior that develops on the rotating-cone with a half-angle $\psi=40^{\circ}$ and then extend the analysis to the larger spectrum of rotating-cone boundary layers.

TABLE II. Critical azimuthal mode numbers $n_{c}$ for the appearance of globally unstable disturbances. Values $n_{c, H}$ are predictions based on coupling solutions of the Ginzburg-Landau equation with numerical simulations of the homogeneous flow, while $n_{c, I}$ represents the parameter range inferred by disturbance development to the inhomogeneous flow.

\begin{tabular}{lcc}
\hline \hline$\psi$ & $n_{c, H}$ & $n_{c, I}$ \\
\hline $20^{\circ}$ & 26 & $20-30$ \\
$30^{\circ}$ & 39 & $30-50$ \\
$40^{\circ}$ & 51 & $50-60$ \\
$50^{\circ}$ & 61 & $60-70$ \\
$60^{\circ}$ & 70 & $70-80$ \\
$70^{\circ}$ & 76 & $70-90$ \\
$80^{\circ}$ & 80 & $80-90$ \\
$90^{\circ}$ & 83 & $80-90$ \\
\hline \hline
\end{tabular}




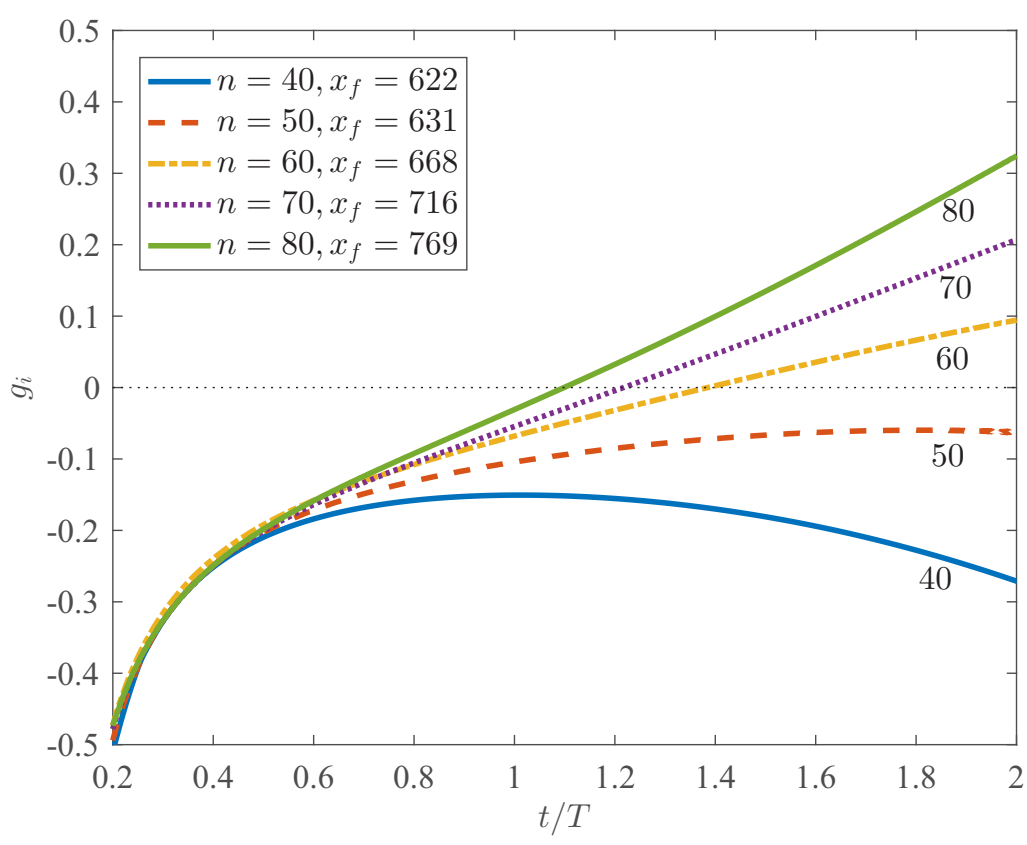

FIG. 4. Temporal growth rates $g_{i}$ as functions of time, for disturbances impulsively excited about the location $x_{f}$ for critical absolute instability, for $n \in[40: 10: 80]$ and $\psi=40^{\circ}$.

\section{A. Cone half-angle $\psi=40^{\circ}$}

Several perturbations to the rotating-cone with a half-angle $\psi=40^{\circ}$ were impulsively excited for azimuthal mode numbers $n \in[40: 80]$ at intervals $\Delta n=10$ about the matching streamwise location $x_{f}$ for critical absolute instability (based on the homogeneous flow approximation). The value for $x_{f}$ associated with each azimuthal mode number is listed within the legend of Fig. 4, which depicts the corresponding growth rates $g_{i}$ as functions of time $t / T$. The time variation has been scaled on the cone rotation rate $T=2 \pi \mathrm{Re}$. Although not included in Fig. 4 or any of the subsequent illustrations, temporal frequencies $g_{r}$ were found to vary in time and along the streamwise $x$ direction. Thus, much like the earlier investigations on the infinite rotating-disk $[9,15,20]$, no fixed global frequencies were obtained, at least for the time duration that the computational results could be accurately relied upon.

The growth rate nearest the bottom of Fig. 4 corresponds to the disturbance generated for $n=$ 40 and $x_{f}=622(\mathrm{Re}=400)$. For the time duration shown, this particular growth rate is always negative and is decreasing after the first period of rotation (i.e., $t / T>1$ ). Thus, the disturbance exhibits strong temporal decay. For larger azimuthal mode numbers $n$, the growth rate is shifted vertically upwards in ascending order of $n$. For $n=50$, the growth rate is again negative for the entire time period plotted, but is less negative than that illustrated for $n=40$. Indeed, towards the end of the second period of rotation, the growth rate (associated with $n=50$ ) appears to approach a fixed (negative) constant. However, it is worth noting that if the disturbance development was extended to larger time, the growth rate might eventually follow the trend displayed for $n=40$ and begin to decrease. Nevertheless, there is clearly a marked difference between the solutions obtained for $n=40$ and 50 .

For those larger azimuthal mode numbers $(n \geqslant 60)$ modeled in Fig. 4, positive increasing temporal growth rates are realized. These particular growth rates increase over the full time illustrated, with little to suggest that they will reverse direction at some later point in time. Furthermore, growth rates increase more rapidly as $n$ is raised to larger values, establishing strongly unstable characteristics. Thus, the global linear stability behavior is similar to that found by Thomas and Davies [15] for 
(a)

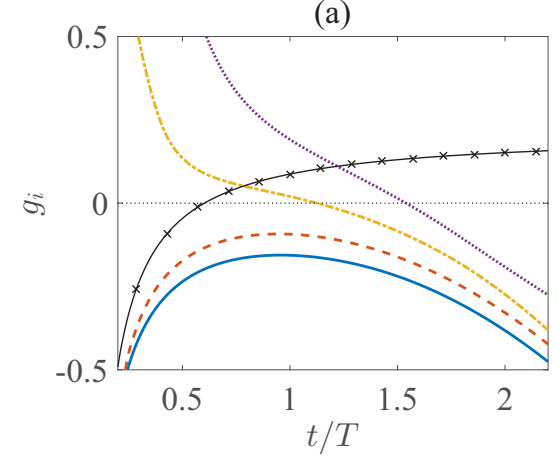

(c)

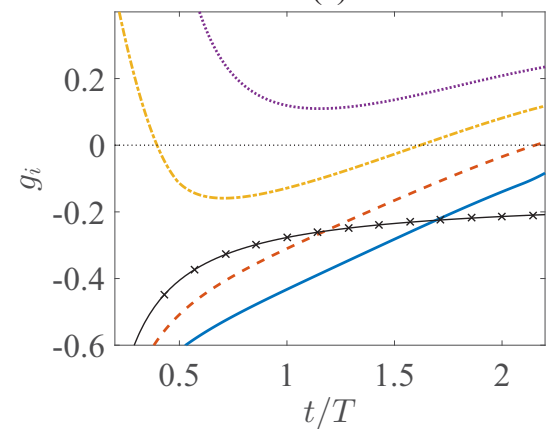

(b)

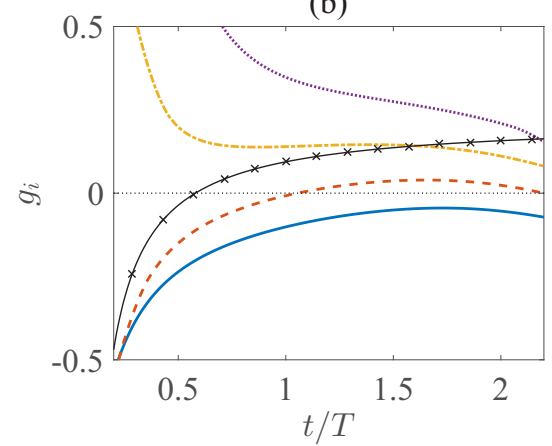

(d)

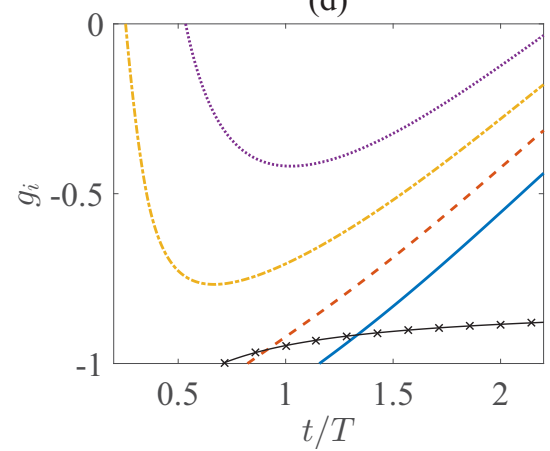

FIG. 5. Temporal growth rates $g_{i}$ for disturbances impulsively excited about $x_{f}=650(\operatorname{Re}=418)$ and for $\psi=40^{\circ}$. Development plotted about $x=x_{f}-25$ (solid lines), $x=x_{f}$ (dashed), $x=x_{f}+25$ (chain) and $x=x_{f}+50$ (dotted), while the solid-crossed lines depict the corresponding solutions in the homogeneous flow about the impulse center. (a) $n=40$, (b) $n=50$, (c) $n=60$, (d) $n=70$.

the infinite rotating-disk (see Fig. 9 of their paper). Positive increasing temporal growth and a form of globally instability develops for sufficiently high azimuthal mode numbers. However, it should be mentioned that the streamwise location associated with these larger azimuthal mode numbers is significantly greater than that corresponding to critical absolute instability $\left(n_{a}=44\right.$ and $x_{a} \approx 627$ for $\psi=40^{\circ}$ ). Additionally, a change in the global response (stable to unstable) appears for values of $n$ that are broadly comparable with the predictions presented in the preceding section that were based on solutions of the linearized Ginzburg-Landau equation. The Ginzburg-Landau model predicts that disturbances will become globally unstable for $n \gtrsim 51$, while those results displayed in Fig. 4 suggest that a change in the global linear response arises for $n \in[50: 60]$. Thus, a reasonable estimate for the appearance of global linear instability is achieved by coupling the Ginzburg-Landau equation with the local stability properties of the homogeneous flow.

Further perturbations were numerically simulated to offer additional support to the argument that global linear instability develops for large enough azimuthal mode numbers. Figure 5 depicts the temporal growth rates associated with four disturbances impulsively excited about $x_{f}=650$. The azimuthal mode number in each instance is increased from $n=40$ to 70 at ten unit intervals. Temporal growth rates are plotted about the impulse center $x_{f}$ and for three additional streamwise positions, located upstream and downstream of $x_{f}$. The solutions established about the impulse center for the corresponding homogeneous flow are plotted within each illustration using a solidcrossed line, where $\operatorname{Re} \equiv x_{f} \sin \psi=418$. Growth rates associated with the homogeneous flow asymptote towards fixed values as time increases. Positive growth and absolute instability are achieved for disturbances $n=40$ and 50, while for the two larger azimuthal mode numbers negative 
temporal growth and at most a convective form of instability develops. However, for the genuine spatially dependent flow, growth rates behave very differently. First, growth rates vary in time and with the streamwise location, which is to be expected given the observations of Fig. 4 and the similarities between the current study and those investigations undertaken by Davies and co-workers $[9,15,21-23]$. Second, there is contrasting behavior in the long-term response. For $n=40$ and 50, growth rates are decreasing, and in the former instance negative growth rates are eventually realized. Furthermore, given the trend of those curves plotted in Fig. 5(b), negative growth rates and temporal decay might be expected to emerge within the next period of rotation. On the other hand, rapidly increasing growth rates are observed for those disturbances generated for $n=60$ and 70, which are significantly greater than those growth rates obtained via the matching homogeneous flow analysis. For $n=60$ and the time duration shown, positive growth rates are attained at all but one of the given streamwise locations, and it is expected that temporal growth will soon be realized about $x=x_{f}-25$ (solid line). Although growth rates remain negative for $n=70$ over the full time period shown, the trajectories of those plots depicted in Fig. 5(d) suggest that positive temporal growth might be expected at all streamwise locations considered, within one or two periods.

The streamwise variation of the temporal growth rate $g_{i}$, that is computed by measuring variations in $g_{i}$ about a fixed point in time, is considerably greater for those results presented for $n=70$. This last observation was also documented by Thomas and Davies [15] for disturbances in the infinite rotating-disk boundary layer and via solutions to the Ginzburg-Landau equation. The flow became globally unstable once the streamwise variation of the temporal growth rate, $\partial g_{i} / \partial x$, was sufficiently large, which arose for azimuthal mode numbers $n$ greater than the conditions for critical absolute instability. Indeed, for those $\rho$ calculations plotted in Fig. 3, $\partial g_{i} / \partial x$ increases with the azimuthal mode number, eventually becoming large enough to bring about a positive valued $\rho$ and unstable behavior.

Figure 6 displays the spatial-temporal disturbance development corresponding to those perturbations illustrated in Fig. 5. The disturbance evolution is depicted in the streamwise-temporal plane using contours of $\left|\omega_{\theta, w}\right|$, where solutions have been normalized about $t=0.2 T$. Additionally, contour levels are drawn using a logarithmic scaling,

$$
N=\ln \left|\omega_{\theta, w}\right| \text {. }
$$

The leading and trailing edges of the four disturbance wave packets are identified as those outer contours labeled $N=-1$ that originate from the initial impulse centered about $x_{f}=650$. In each instance, the leading edge propagates downstream with approximately the same nonzero velocity. However, the four trailing edges display very contrasting features, bringing about very different forms of global linear stability. For $n=40$, convectively unstable characteristics appear to dominate the impulse response, as the trailing edge propagates downstream, with what appears to be an increasing velocity. As the azimuthal mode number is increased to larger values, $n=60$ and 70 , the trailing edge can be seen to reverse direction and propagate upstream as it approaches the streamwise location $x_{a}$ for the onset of absolute instability associated with that particular azimuthal mode number [represented by vertical dashed lines in Fig. 6(c) and 6(d)]. Given the wave-packet formations depicted for the two latter perturbations, and their corresponding temporal growth rates plotted in Fig. 5, it would appear that a form of global instability is emerging.

Two more disturbances were impulsively excited about $x_{f}=700$, for $n=40$ and 60 . The spatialtemporal disturbance wave packets and matching temporal growth rates for these two parameter settings are illustrated in Fig. 7. The line types for the growth rates are the same as those described in Fig. 5. Absolute instability is clearly demonstrated in both instances when the homogeneous flow approximation is enforced, as positive temporal growth is obtained at large time (solid-crossed lines). Meanwhile, growth rates corresponding to those disturbances to the inhomogeneous flow are decreasing for $n=40$ and increasing for $n=60$. Temporal decay is observed about all streamwise positions for the smaller modal disturbance, while strong increasing temporal growth is obtained for the larger azimuthal mode number. The two disturbance wave packets plotted in Figs. 7(a) and 7(b) further illustrate the differences in global behavior that are realized for variable $n$. The 
(a)

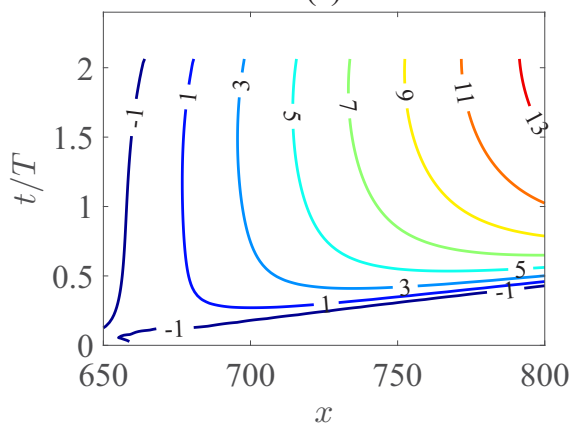

(c)

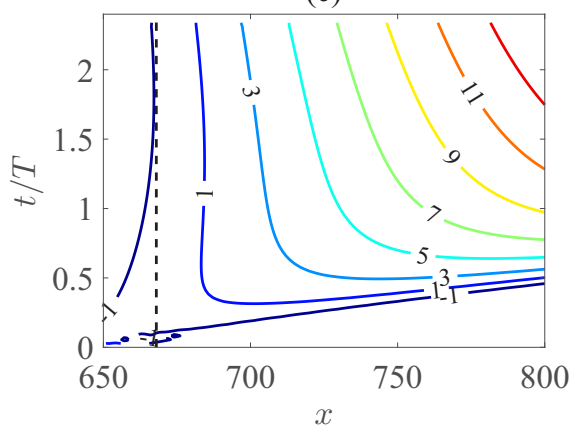

(b)

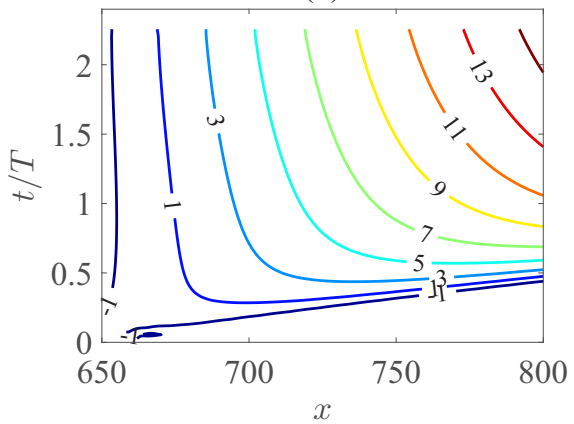

(d)

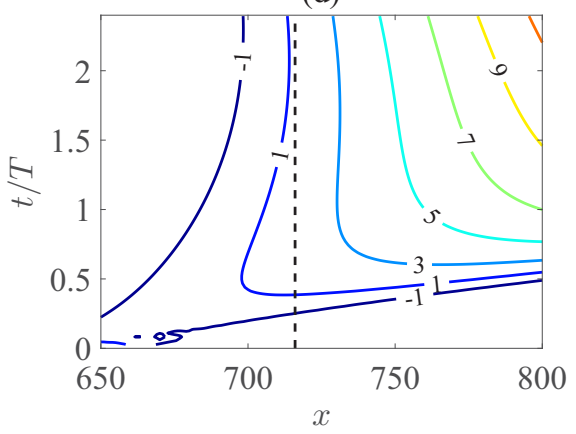

FIG. 6. Spatial-temporal disturbance development of the azimuthal vorticity on the disk surface $\left|\omega_{\theta, w}\right|$, for an impulsive center about $x_{f}=650(\operatorname{Re}=418)$ and for $\psi=40^{\circ}$. (a) $n=40$, (b), (c) $n=60$, (d) $n=70$. Vertical dashed lines in (c) and (d) represent the streamwise location $x_{a}$ for the onset of absolute instability, associated with the given azimuthal mode numbers.

trailing edge associated with the disturbance for $n=40$ initially propagates upstream, but eventually reverses direction and appears to be moving downstream by the end of the given time period. On the other hand, the trailing edge matching to $n=60$ propagates upstream for the entire time duration shown and global linear instability develops. Hence, numerical simulations provide a convincing and compelling argument for the appearance of globally unstable characteristics at high azimuthal mode numbers.

\section{B. Cone half-angles $\psi \in\left[20^{\circ}: 80^{\circ}\right]$}

The above analysis was extended to a further six rotating-cone geometries, with half-angles $\psi \in$ $\left[20^{\circ}: 80^{\circ}\right.$ ]. Figure 8 reproduces the earlier analysis presented in Fig. 4 for $\psi=40^{\circ}$, comparing temporal growth rates associated with disturbances generated for several combinations of the cone half-angle $\psi$ and azimuthal mode number $n$ (listed within each legend and the caption of Fig. 8). Each perturbation was impulsively excited about the streamwise location $x_{f}$ (given within each legend) that matches the respective onset of absolute instability. For each value of $\psi$ modeled, growth rates display similar trends. For relatively small $n$, growth rates decrease and disturbances decay. However, for larger $n$, strong temporal growth and a form of global instability develops. The azimuthal mode number $n$ required to bring about a change in the global response (stable to unstable) decreases with smaller cone half-angles. Furthermore, numerical simulations suggest that the critical value $n_{c}$ necessary to engineer global linear instability is broadly comparable with the earlier predictions obtained by coupling local stability results with the Ginzburg-Landau equation. For instance, the Ginzburg-Landau model suggests that for $\psi=60^{\circ}, n \gtrsim 70$ was sufficient to bring 
(a)

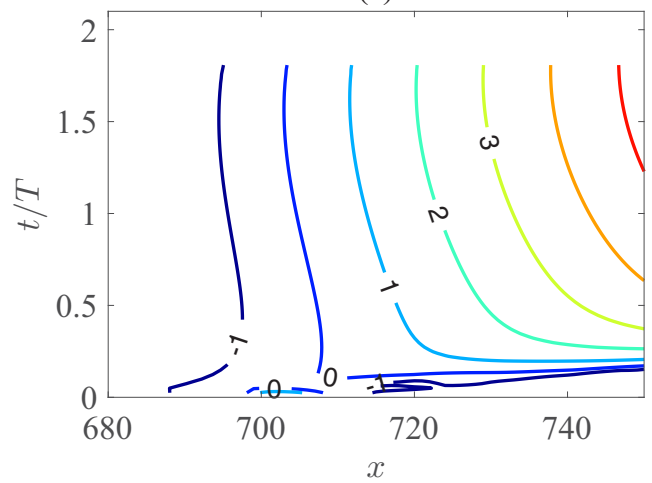

(c)

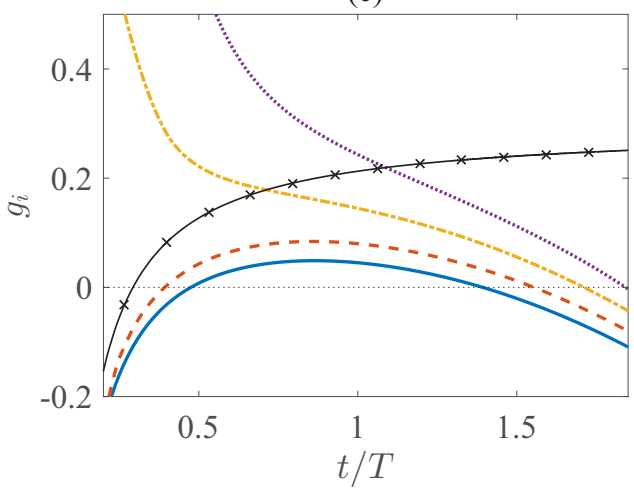

(b)

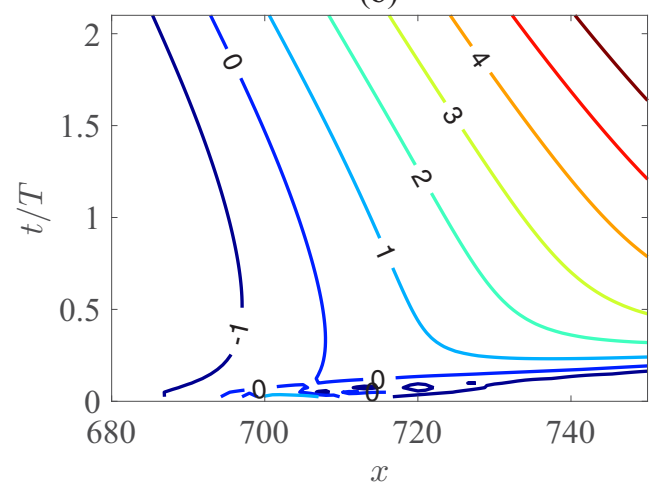

(d)

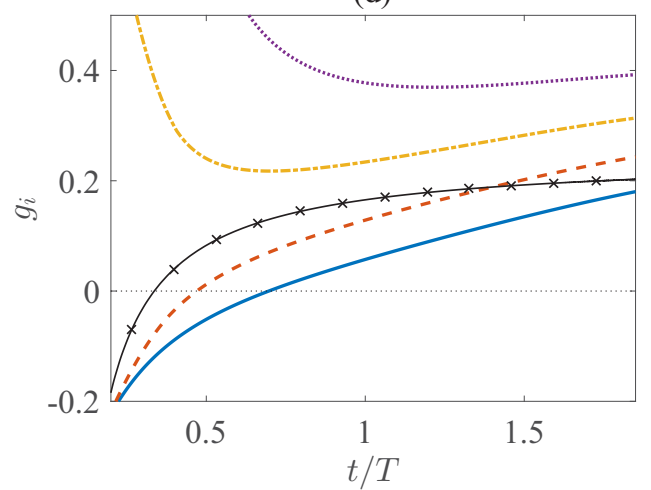

FIG. 7. Spatial-temporal disturbance wave packets and matching temporal growth rates for an impulse centered about $x_{f}=700(\operatorname{Re}=450)$ for $\psi=40^{\circ}$. (a) and (c): $n=40$; (b) and (d) $n=60$. Line types for growth rates in (c) and (d) are the same as those given in Fig. 5.

about globally unstable behavior, while numerical simulations [Fig. 8(c)] infer that global instability emerges for $n \in[70: 80]$. Additionally, for $\psi=20^{\circ}, n \gtrsim 26$ was predicted as being necessary for global linear instability, while results plotted in Fig. 8(f) suggest a change in global behavior is realized for $n \in$ [20:30]. Similar comparisons were also obtained for the remaining four rotatingcones investigated, and results are included in Table II for comparison.

\section{CONCLUSIONS}

The global linear stability of the family of rotating-cone boundary layers has been investigated for an extensive range of cone half-angles and flow parameter settings. The cones were rotated within a quiescent medium and modeled as infinitely long in the streamwise $x$ direction. A localised impulsive wall forcing, implemented via a linearization of the no-slip condition, was used to excite perturbations and study their evolution. For those numerical simulations established using a homogeneous flow approximation (whereby the streamwise dependence of the basic state was ignored), solutions were found to be in excellent agreement with local linear stability results that utilize the same flow assumptions [8].

Disturbances to the genuine spatially varying inhomogeneous flow were found to display global stability characteristics qualitatively similar to that observed in the infinite rotating-disk boundary layer. For azimuthal mode numbers below a critical threshold $n_{c}$, the flow was globally stable; disturbance evolution was dominated by convective characteristics that were similar in appearance 
(a)

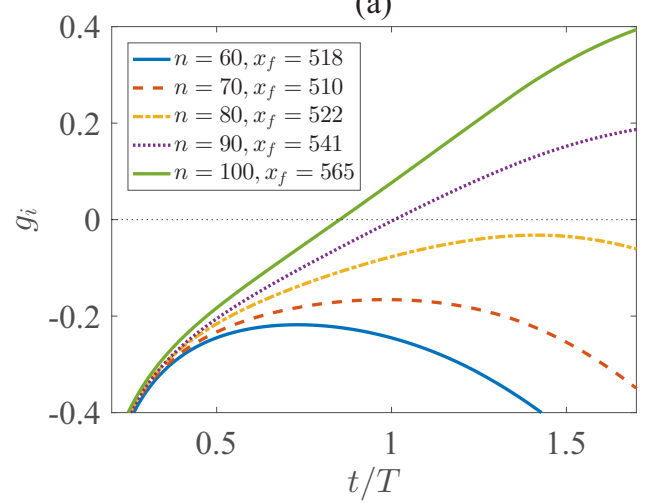

(c)

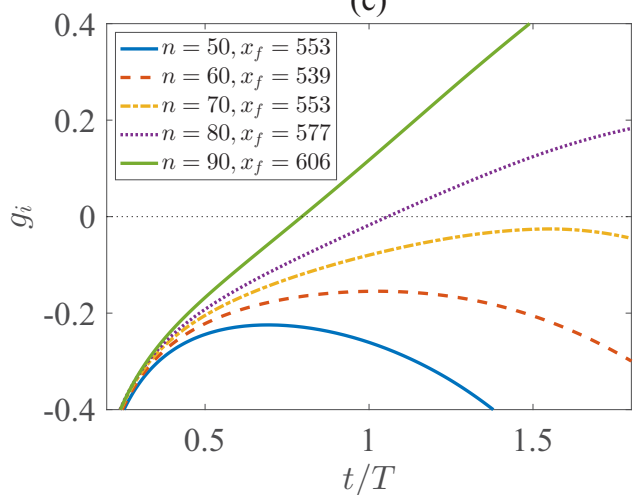

(e)

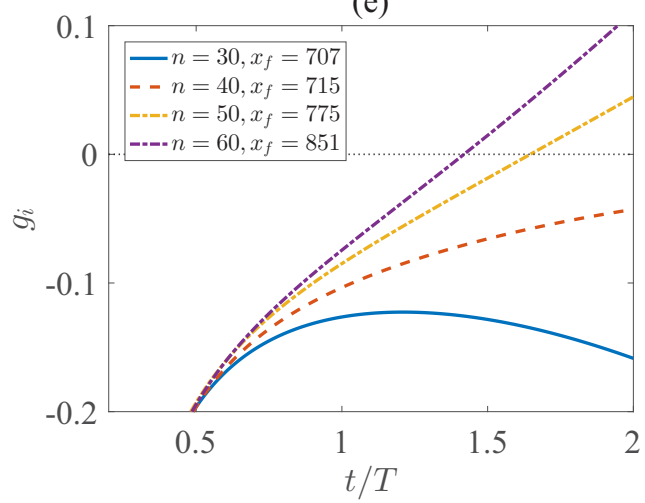

(b)

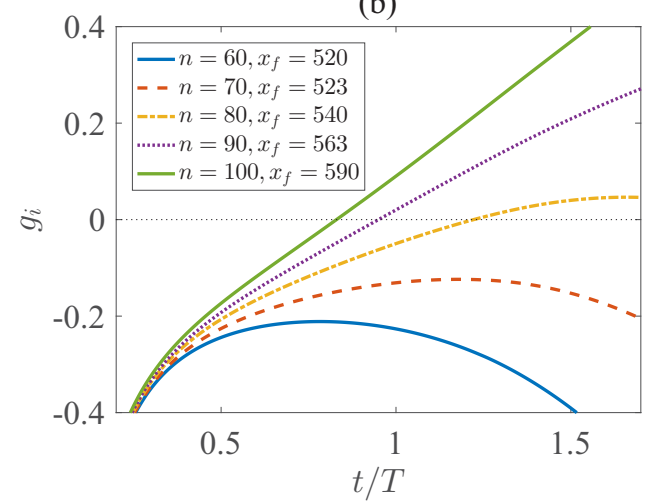

(d)

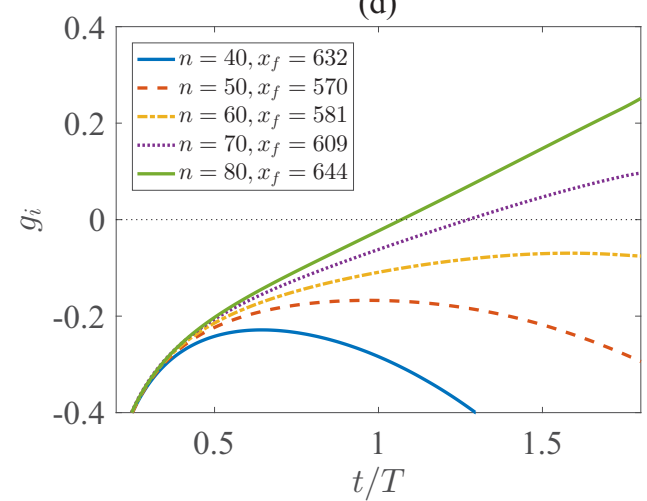

(f)

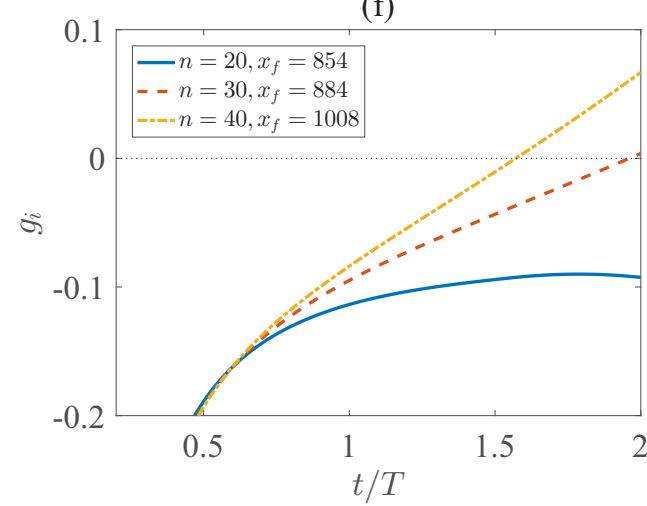

FIG. 8. Temporal growth rates $g_{i}$, for disturbances impulsively excited about the onset of absolute instability $x_{f}$ for variable $n$. (a) $\psi=80^{\circ}$, (b) $\psi=70^{\circ}$, (c) $\psi=60^{\circ}$, (d) $\psi=50^{\circ}$, (e) $\psi=30^{\circ}$, (f) $\psi=20^{\circ}$.

to that observed by Davies and Carpenter [9]. However, for larger azimuthal mode numbers, disturbances displayed features matching the recent observations of Thomas and Davies [15]; a form of global instability was realized that is characterized by a faster than exponential temporal growth. In all instances modeled, the azimuthal mode number $n_{c}$ required for a change in the global response was greater than the critical conditions for absolute instability.

The azimuthal mode number $n_{c}$ required for a change in the global behavior was predicted by coupling solutions of the linearized Ginzburg-Landau equation with the local stability properties of 
disturbances to the homogeneous flow. Radial variations in the temporal growth rate were found to increase for larger azimuthal mode numbers, eventually becoming sufficient to bring about global linear instability. For all rotating-cone systems studied, the predictions for $n_{c}$ were reasonably consistent with the numerical simulation results obtained for the inhomogeneous flow. Globally unstable disturbances are thus found in many rotating boundary layers. The local-global linear stability properties are similar to that outlined in Huerre and Monkewitz [19], whereby a region of local absolute instability is a necessary but not sufficient condition for global instability to develop.

Although the above study clearly demonstrates that globally unstable disturbances can develop in the infinite rotating-cone and -disk boundary layers, it remains to be seen what effect this has on the laminar-turbulent transition process in real-life experiments. Using direct numerical simulations of the rotating-disk with disturbances excited via finite roughness elements, Appelquist et al. [42] found that convective instabilities were the primary mechanism for the onset of transition to turbulence. Indeed, Thomas and Davies [15] concluded that the high azimuthal modal disturbances were very unlikely to be observed in experimental studies of the rotating-disk, due to the huge spatial growth associated with the convective crossflow instabilities. Moreover, recent investigations [11-14] that modelled finite disk effects, suggest that globally unstable disturbances can be created at lower azimuthal mode numbers than that necessary herein. Thus, there remain several questions for the future study of rotating boundary layers. Do the high $n$ azimuthal mode numbers feature in the transition process and what are the implications for finite cone or disk models?

[1] R. J. Lingwood, Absolute instability of the boundary-layer on a rotating-disk, J. Fluid Mech. 299, 17 (1995).

[2] Th. von Kármán, Über laminare und turbulente reibung, Z. Angew. Math. Mech. 1, 233 (1921).

[3] R. J. Briggs, Electron-Stream Interactions in Plasmas (MIT Press, Cambridge, MA, 1964).

[4] N Gregory, J. T. Stuart, and W. S. Walker, On the stability of three-dimensional boundary-layers with application to the flow due to a rotating disk, Philos. Trans. R. Soc. London, Ser. A 248, 155 (1955).

[5] L. M. Mack, The wave pattern produced by point source on a rotating disk, AIAA Paper 85-0490 (1985).

[6] S. J. Garrett and N. Peake, The stability and transition of the boundary layer on a rotating sphere, J. Fluid Mech. 456, 199 (2002).

[7] S. J. Garrett and N. Peake, The stability of the boundary layer on a sphere rotating in a uniform axial flow, Eur. J. Mech. B 23, 241 (2004).

[8] S. J. Garrett and N. Peake, The absolute instability of the boundary layer on a rotating cone, Eur. J. Mech. B 26, 344 (2007).

[9] C. Davies and P. W. Carpenter, Global behaviour corresponding to the absolute instability of the rotatingdisc boundary layer, J. Fluid Mech. 486, 287 (2003).

[10] C. Davies and P. W. Carpenter, A novel velocity-vorticity formulation of the Navier-Stokes equations with applications to boundary layer disturbance evolution, J. Comput. Phys. 172, 119 (2001).

[11] E. Appelquist, P. Schlatter, P. H. Alfredsson, and R. J. Lingwood, On the global nonlinear instability of the rotating-disk flow over a finite domain, J. Fluid Mech. 803, 332 (2016).

[12] J. J. Healey, Model for unstable global modes in the rotating-disk boundary layer, J. Fluid Mech. 663, 148 (2010).

[13] E. Appelquist, P. Schlatter, P. H. Alfredsson, and R. J. Lingwood, Global linear instability of the rotatingdisk flow investigated through simulations, J. Fluid Mech. 765, 612 (2015).

[14] E. Appelquist, P. Schlatter, P. H. Alfredsson, and R. J. Lingwood, Investigation of the global instability of the rotating-disk boundary layer, Proc. IUTAM 14, 321 (2015).

[15] C. Thomas and C. Davies, On the impulse response and global instability development of the infinite rotating-disc boundary layer, J. Fluid Mech. 857, 239 (2018). 
[16] R. J. Lingwood, An experimental study of absolute instability of the rotating-disk boundary-layer flow, J. Fluid Mech. 314, 373 (1996).

[17] H. Othman and T. C. Corke, Experimental investigation of absolute instability of a rotating-disk boundarylayer, J. Fluid Mech. 565, 63 (2006).

[18] R. J. Lingwood and P. H. Alfredsson, Instabilities of the von Kármán boundary layer, Appl. Mech. Rev. 67, 030803 (2015).

[19] P. Huerre and P. A. Monkewitz, Local and global instabilities in spatially developing flows, Annu. Rev. Fluid Mech. 22, 473 (1990).

[20] C. Davies, C. Thomas, and P. W. Carpenter, Global stability of the rotating disc boundary layer, J. Eng. Math. 57, 219 (2007).

[21] C. Thomas and C. Davies, The effects of mass transfer on the global stability of the rotating-disk boundary layer, J. Fluid Mech. 663, 401 (2010).

[22] C. Thomas and C. Davies, Global stability of the rotating-disc boundary layer with an axial magnetic field, J. Fluid Mech. 724, 510 (2013).

[23] C. Davies and C. Thomas, Global stability behaviour for the BEK family of rotating boundary layers, Theor. Comput. Fluid Dyn. 31, 519 (2017).

[24] R. E. Hunt and D. G. Crighton, Instability of flows in spatially developing media, Proc. R. Soc. London, Ser. A 435, 109 (1991).

[25] A. M. Soward, On the finite amplitude thermal instability of a rapidly rotating sphere, Geophys. Astrophys. Fluid Dyn. 9, 19 (1977).

[26] A. M. Soward, Thin disc kinematics $\alpha \omega$-dynamo models: II. Short length scale modes, Geophys. Astrophys. Fluid Dyn. 64, 201 (1992).

[27] D. Harris, A. P. Bassom, and A. M. Soward, An inhomogeneous Landau equation with application to spherical Couette flow in the narrow gap limit, Physica D 137, 260 (2000).

[28] F. Kreith, D. Ellis, and J. Giesing, An experimental investigation of the flow engendered by a rotating cone, Appl. Sci. Res. A 11, 430 (1962).

[29] C. L. Tein and D. T. Campbell, Heat and mass transfer from rotating cones, J. Fluid Mech. 17, 105 (1963).

[30] F. Salzberg and S. P. Kezios, Mass transfer from a rotating cone in axisymmetric flow, J. Heat Transfer 87, 469 (1965).

[31] R. Kappesser, R. Greif, and I. Cornet, Mass transfer on rotating cones, Appl. Sci. Res. 28, 442 (1973).

[32] R. Kobayashi and H. Izumi, Boundary-layer transition on a rotating cone in still fluid, J. Fluid Mech. 127, 353 (1983).

[33] R. Kobayashi, Y. Kohama, and M. Kurosawa, Boundary-layer transition on a rotating cone in axial flow, J. Fluid Mech. 127, 341 (1983).

[34] Y. Kohama, Behaviour of spiral vortices on a rotating cone in axial flow, Acta Mech. 51, 105 (1984).

[35] R. Kobayashi, Linear stability theory of boundary layer along a cone rotating in axial flow, Bull. Japan Soc. Mech. Eng. 24, 934 (1981).

[36] S. J. Garrett, Z. Hussain, and S. O. Stephen, Boundary-layer transition on broad cones rotating in an imposed axial flow, AIAA J. 48, 1184 (2010).

[37] S. J. Garrett, Linear growth rates of types I and II convective modes within the rotating-cone boundary layer, Fluid Dyn. Res. 42, 025504 (2010).

[38] S. J. Garrett, Z. Hussain, and S. O. Stephen, The cross-flow instability of the boundary layer on a rotating cone, J. Fluid Mech. 622, 209 (2009).

[39] Z. Hussain, S. J. Garrett, and S. O. Stephen, The centrifugal instability of the boundary-layer flow over slender rotating cones, J. Fluid Mech. 755, 274 (2014).

[40] Z. Hussain, S. J. Garrett, S. O. Stephen, and P. T. Griffiths, The centrifugal instability of the boundary-layer flow over a slender rotating cone in an enforced axial free stream, J. Fluid Mech. 788, 70 (2016).

[41] W. Mangler, Boundary layers on bodies of revolution in symmetrical flow, Ber. Aerodyn Versuchsanst. Goett., Report 45/A/17 (1945).

[42] E. Appelquist, P. Schlatter, P. H. Alfredsson, and R. J. Lingwood, Transition to turbulence in the rotatingdisk boundary-layer flow with stationary vortices, J. Fluid Mech. 836, 43 (2018). 